\title{
Specify Other HPV Typing Method
}

National Cancer Institute

\section{Source}

National Cancer Institute. Specify Other HPV Typing Method. NCI Thesaurus. Code C162471.

A directive to specify the method of HPV typing. 